\title{
Motor cortex transcriptome reveals microglial key events in amyotrophic lateral sclerosis
}

Oriol Dols-Icardo, PhD, Víctor Montal, MSc, Sònia Sirisi, PhD, Gema López-Pernas, MSc,

Laura Cervera-Carles, PhD, Marta Querol-Vilaseca, MSc, Laia Muñoz, BSc, Olivia Belbin, PhD, Daniel Alcolea, MD, PhD, Laura Molina-Porcel, MD, PhD, Jordi Pegueroles, MSc, Janina Turón-Sans, MD, Rafael Blesa, MD, PhD, Alberto Lleó, MD, PhD, Juan Fortea, MD, PhD, Ricard Rojas-García, MD, PhD, and Jordi Clarimón, PhD

Neurol Neuroimmunol Neuroinflamm 2020;7:e829. doi:10.1212/NXI.0000000000000829

\section{Abstract}

\section{Objective}

To identify transcriptomic changes, neuropathologic correlates, and cellular subpopulations in the motor cortex of sporadic amyotrophic lateral sclerosis (ALS).

\section{Methods}

We performed massive RNA sequencing of the motor cortex of patients with ALS $(n=11)$ and healthy controls (HCs; $\mathrm{n}=8$ ) and analyzed gene expression alterations, differential isoform usage, and gene coexpression networks. Furthermore, we used cell type deconvolution algorithms with human single-nucleus RNA sequencing data as reference to identify perturbations in cell type composition associated with ALS. We performed immunohistochemical techniques to evaluate neuropathologic changes in this brain region.

\section{Results}

We report extensive RNA expression alterations at gene and isoform levels, characterized by the enrichment of neuroinflammatory and synaptic-related pathways. The assembly of gene coexpression modules confirmed the involvement of these 2 major transcriptomic changes, which also showed opposite directions related to the disease. Cell type deconvolution revealed an overrepresentation of microglial cells in ALS compared with HC. Notably, microgliosis was driven by a subcellular population presenting a gene expression signature overlapping with the recently described disease-associated microglia (DAM). Using immunohistochemistry, we further evidenced that this microglial subpopulation is overrepresented in ALS and that the density of pTDP43 aggregates negatively correlates with the proportion of microglial cells.

\section{Conclusions}

DAM has a central role in microglia-related neuroinflammatory changes in the motor cortex of patients with ALS, and these alterations are coupled with a reduced expression of postsynaptic transcripts.

\author{
Correspondence \\ Dr. Clarimón \\ jclarimon@santpau.cat \\ or Dr. Dols-Icardo \\ odols@santpau.cat
}




\section{Glossary}

ALS = amyotrophic lateral sclerosis; DAM = disease-associated microglia; FFPE = formalin-fixed paraffin-embedded; GO = gene ontology; HC = healthy control; IHC = immunohistochemistry; KEGG = Kyoto Encyclopedia of Genes and Genomes; MuSiC = Multi-Subject Single Cell deconvolution; NTB = Neurological Tissue Bank; qPCR = quantitative real-time PCR; RIN = RNA integrity; RNAseq $=$ RNA sequencing; ROI = region of interest ROSMAP $=$ Religious Orders Study and Memory and Aging Project; snRNAseq = single-nucleus RNAseq; TREM2 = triggering receptor expressed on myeloid cells 2; WGCNA = weighted gene coexpression network analyses.

Amyotrophic lateral sclerosis (ALS) is a neurodegenerative disease neuropathologically characterized by the aberrant cytoplasmic aggregation and phosphorylation of the $43-\mathrm{kDa}$ transactive response DNA-binding protein (pTDP43) in the majority of ALS cases at postmortem evaluation, which is known to occur in a sequential manner starting in the motor cortex. ${ }^{1}$ Although the causes that lead to nongenetic forms of ALS (sporadic ALS) have yet to be fully determined, the presence of neuroinflammation is a consistent feature in the CNS of affected patients. ${ }^{2}$ Microglia are macrophage-like innate immune cells of the CNS that, as a result of disease conditions, change their gene expression profile to adapt and acquire a reactive state. ${ }^{3}$ Recently, a study using single-cell RNA sequencing from the CNS of Alzheimer disease and ALS mouse models reported that a specific microglial subpopulation, known as diseaseassociated microglia (DAM), was the cell type responsible for microgliosis and that triggering receptor expressed on myeloid cells 2 (TREM2) is a major driver of DAM activation. ${ }^{4}$ In support of this discovery, some chitinase proteins, which are known markers of microglial activation, ${ }^{5,6}$ have been consistently shown to be increased in the CSF of patients with ALS.,

The pivotal role of TDP43 and other genes known to cause ALS (such as FUS, hnRNPA2B1, hnRNPA1, TAF15 or TIA1) in the metabolism of RNA has implicated RNA dyshomeostasis as a crucial event in the pathophysiology of the disease. ${ }^{9}$ An unbiased resource to obtain a comprehensive signature of gene and isoform expression changes associated with the whole tissue response to disease conditions is total RNA sequencing (RNAseq) in bulk tissue. Furthermore, the advent of novel sequencing tools such as single-nucleus RNAseq (snRNAseq) has opened a new window to better explore the transcriptome at a cell level and has unraveled a complex and huge variety of human cell types with unique expression profiles in the human brain. ${ }^{10-12}$ These transcriptomic signatures can be applied to perform cell type deconvolution of bulk RNAseq data using novel deconvolution methods. These methods take into account cross-subject and cross-cell variability of gene expression profiles, without relying on preselected markers, thereby providing more realistic cell type proportion estimations and yielding crucial information about the cellular heterogeneity that is associated with a pathologic condition. ${ }^{13}$
The brain motor cortex is affected at the most early stages of the disease and is one of the most vulnerable regions in ALS. However, studies of this critical region both at the transcriptional and immunohistochemical level are lacking. In the present work, we aim to characterize the motor cortex of sporadic ALS cases through total RNAseq analyses. We also use cell type deconvolution using human snRNAseq data as reference and immunohistochemical analyses to resolve a signature of neuropathologic changes associated with ALS in this particular brain region.

\section{Methods}

\section{Human samples}

The study included human motor cortex (Brodmann area 4) samples provided by the Neurological Tissue Bank (NTB) of the Biobanc-Hospital Clínic-IDIBAPS. None of the brain tissues presented any infarcts in the motor cortex. Diagnosis of ALS complied with the El Escorial criteria during life, ${ }^{14}$ and none of the patients had a family history of ALS or dementia, nor did show any sign of cognitive impairment. All patients presented PTDP43 inclusions in the motor cortex at postmortem examination. None of the samples carried the C9orf72 hexanucleotide repeat expansion or mutations in the $T B K 1$ gene, the most common genetic causes related to adultonset ALS in Spain. ${ }^{15,16}$

\section{RNA extraction and sequencing}

Using a mortar and liquid nitrogen, the tissue $(60 \mathrm{mg})$ was grinded to powder and transferred to a solution of $600 \mu \mathrm{L}$ of TRIzol reagent (Thermo Fisher Scientific, Waltham, MA). We used standard recommendations and procedures to extract RNA with TRIzol, the RNeasy Mini Kit (Qiagen, Hilden, Germany), and the Rnase-Free Dnase Set (Qiagen). Qubit was used to measure RNA concentration, whereas RNA integrity (RIN) was verified on an Agilent 2100 Bioanalyzer (Agilent Technologies, Santa Clara, CA). Only samples with a quantity threshold of $5 \mu \mathrm{g}$ and RIN $\geq 6.5$ were used for total RNAseq. The final study group included 11 ALS cases and 8 healthy controls (HCs) (table 1). Paired-end sequencing libraries were prepared using the TruSeq Stranded Total RNA Library Preparation kit (Illumina, San Diego, CA) and sequenced on the Illumina HiSeq 2500 platform by the Centro Nacional de Análisis Genómicos (CNAG; Barcelona, Spain) with 101-bp paired-end reads to achieve at least 100 million 
Table 1 Demographic, clinical, and quality control data

\begin{tabular}{|c|c|c|c|c|c|c|c|c|}
\hline Sample ID & Group & Age at death, y & Sex & Age at onset, y & Duration, mo & Site of onset & RIN & PMI, h \\
\hline AF5214 & ALS & 64 & Male & 62 & 33 & Limb & 8.2 & 7 \\
\hline AF5215 & ALS & 50 & Male & 46 & 58 & Limb & 8.8 & 13 \\
\hline AF5216 & ALS & 60 & Female & 54 & 72 & Limb & 8.3 & 6 \\
\hline AF5218 & ALS & 53 & Female & 50 & 32 & Limb & 8.4 & 9 \\
\hline AF5220 & ALS & 82 & Male & 81 & 1 & Bulbar & 7.7 & 16 \\
\hline AF5222 & ALS & 54 & Female & 53 & 18 & Limb & 9 & 7 \\
\hline AF5224 & ALS & 70 & Female & 68 & 24 & Limb & 8.4 & 14 \\
\hline AF5227 & ALS & 78 & Male & 76 & 24 & Limb & 8.1 & 12 \\
\hline AF5228 & ALS & 77 & Female & 75 & 31 & Bulbar & 7 & 16 \\
\hline AF5229 & ALS & 57 & Female & 54 & 24 & Limb & 6.8 & 5 \\
\hline AF5232 & ALS & 52 & Male & 51 & 12 & Limb & 7.4 & 18 \\
\hline AF5219 & $\mathrm{HC}$ & 64 & Male & - & - & - & 8.2 & 10 \\
\hline AF5221 & $\mathrm{HC}$ & 78 & Male & - & - & - & 7.9 & 6 \\
\hline AF5226 & $\mathrm{HC}$ & 81 & Female & - & - & - & 7.5 & 23 \\
\hline AF5231 & $\mathrm{HC}$ & 83 & Female & - & - & - & 7.3 & 7 \\
\hline AF5234 & $\mathrm{HC}$ & 58 & Male & - & - & - & 7.5 & 4 \\
\hline AF5235 & $\mathrm{HC}$ & 76 & Male & - & - & - & 7.5 & 11 \\
\hline AF5236 & $\mathrm{HC}$ & 83 & Female & - & - & - & 7.7 & 7 \\
\hline AF5237 & $\mathrm{HC}$ & 68 & Female & - & - & - & 6.5 & 13 \\
\hline
\end{tabular}

Abbreviations: ALS = amyotrophic lateral sclerosis; $\mathrm{HC}=$ healthy control; $\mathrm{PMI}=$ postmortem interval; RIN = RNA integrity number.

paired-end reads for each sample (ranging from 103.447.000 to 138.990 .000 paired-end reads).

\section{Data processing}

We aligned FASTQ files to the Grch38.p12 genome assembly using STAR v2.6.1a. ${ }^{17}$ We then used GATK Best Practices ${ }^{18}$ for variant calling and FeatureCounts (within the Subread v.1.6.2 package $^{19}$ ) to assign fragments to each gene feature included in the Grch38v94 gene transfer format file. For differential isoform usage, we used the STAR v.2.6.1 package in quantMode TranscriptomeSAM mode and used Salmon to obtain isoform expression values. ${ }^{20}$ For differential gene expression and differential isoform usage analyses, we used DESeq2 v1.24, ${ }^{21}$ which applies the Benjamini-Hochberg for $p$ value adjustment. We used a false discovery rate threshold of $5 \%$. To perform gene ontology (GO) and Kyoto Encyclopedia of Genes and Genomes (KEGG) pathway enrichment analyses, we used Metascape 3.0 (metascape.org), ${ }^{22}$ applying default parameters (minimum overlap $=3, p$ value cutoff $=$ 0.01 , and minimum enrichment $=1.5$ ). For the synaptic enrichment analyses, we used the web server SynGO (syngoportal.org/), which provides an expert-curated resource for synaptic function and gene enrichment analysis. ${ }^{23}$

\section{Validation of transcriptome changes by quantitative real-time PCR}

We performed quantitative real-time PCR (qPCR) on the same brain-derived RNA samples used for total RNAseq (11 sALS cases and 8 controls). A total of 500 ng of RNA was used to generate cDNA with the RevertAid First Strand cDNA Synthesis Kit (Thermo Fisher Scientific), as per the manufacturer's instructions. All qPCRs were conducted using Fast SYBR Green Master Mix (Thermo Fisher Scientific) and run on an ABI Prism 7900HT Fast Real-Time PCR System (Applied Biosystems, Foster City, CA). All primer pairs used are listed in table e-1, links.lww.com/NXI/A280. For relative quantification, we applied the $\Delta \Delta \mathrm{Ct}$ method, using RPL13 as an endogenous control to normalize the data. We decided to use RPL13 as a housekeeping gene as it showed the smallest coefficient of variation $(<3.5 \%)$ compared with the 2 other putative housekeeping genes that we assessed (RPLO and GAPDH).

\section{Cell type deconvolution}

We used the recently developed Multi-Subject Single Cell deconvolution $(\mathrm{MuSiC})^{13}$ method to perform cell type deconvolution of our bulk RNAseq data. As a reference, we 
used the most comprehensive human snRNAseq data set available. This data set includes the RNAseq from 70,634 cells in the frontal cortex (Brodmann area 10) of 24 Alzheimer disease cases and $24 \mathrm{HCs}$ from the Religious Orders Study and Memory and Aging Project (ROSMAP), ${ }^{12}$ grouped into 8 major cellular populations (excitatory neurons, inhibitory neurons, microglia, astrocytes, oligodendrocytes, oligodendrocyte precursor cells, endothelial cells, and pericytes). To check consistency and validate our results, we used 2 independent snRNAseq repositories as a reference to estimate major cell type proportions. To this aim, we downloaded the medial temporal gyrus snRNAseq data derived from the Allen Brain Atlas, which includes 15,928 cells from 8 human tissue donors. We selected this data set as it contains double the number of nuclei than the primary visual cortex and the anterior cingulate cortex, also contained in the database. ${ }^{10}$ The third data set comprised 10,319 cells derived from the frontal cortex of 4 individuals. ${ }^{11}$ Cell type deconvolution of cellular subpopulations was performed using the ROSMAP data as reference.

\section{Weighted gene coexpression network analyses}

We accounted for genes with more than 10 counts across all individuals. DESeq2 was then used to normalize the input data to construct coexpression networks using the weighted gene coexpression network analyses (WGCNA) package. ${ }^{24}$ We then constructed the signed weighted correlation network, which takes into account both negative and positive correlations, using the manual function in WGCNA, applying the biweight midcorrelation, selecting a power of 7 (the lowest possible power term where topology fits a scale free network), and run in a single block analysis. We defined all modules by the hybrid treecutting option with deepsplit parameter $=2$, applying a minimal module size of 30 genes and merging modules with a cutHeight $\leq 0.25$. Module eigengenes (first principal component as a summary of each module) summarized the modules and correlations performed using spearman correlation for continuous traits and Pearson correlation for binary traits (bicor and corPvalueStudent, included in the WGCNA package). We used Cytoscape 3.7.2 to visualize networks.

\section{Immunohistochemistry}

We used formalin-fixed paraffin-embedded (FFPE) motor cortex tissue sections of $5 \mu \mathrm{m}$ in 11 patients with ALS and 7 controls. One control sample (AF5237) was excluded from the analysis due to lack of availability of FFPE motor cortex sections. We dewaxed the FFPE sections by placing them in xylene and decreasing concentrations of ethanol, followed by hydration 30 minutes in distilled water. We then treated tissue sections with a solution containing 10\% methanol, 3\% $\mathrm{H}_{2} \mathrm{O}_{2}$, and PBS for 10 minutes to inhibit endogenous peroxidase and boiled in citrate $1 x$ solution to mediate antigen retrieval. Once cooled, we blocked all sections with 5\% bovine serum albumin for 1 hour at room temperature and incubated them overnight with the corresponding primary antibody at $4^{\circ} \mathrm{C}$ and then with the anti-rabbit horse radish peroxidase secondary antibody $(1: 200)$ for 1 hour at room temperature. Primary antibodies were Ibal rabbit polyclonal (1:500; Wako Chemicals, Osaka, Japan) and ps409/ 410-TDP rabbit polyclonal (1:2000; Cosmo Bio, Tokyo, Japan). Following peroxidase development with 3,3'-diaminobenzidine tetrahydrochloride (Dako liquid $\mathrm{DAB}+$ substrate Chromogen System; Dako Denmark, Glostrup, Denmark), sections were stained with hematoxylin (EnVision FLEX Haematoxylin; Dako Denmark) and dehydrated using increasing concentrations of ethanol and then mounted with DPX mounting medium (PanReac AppliChem, ITW Reagents, Chicago, IL). For immunofluorescence, we used 3 ALS and 3 control motor cortex sections, which were incubated with primary antibodies overnight at $4^{\circ} \mathrm{C}$. We used Ibal rabbit polyclonal antibody as a marker for the broad microglial population (1:500; Wako Chemicals) and human HLA-DP, DQ, DR antigen (MHC class II markers), mouse clone CR3/43 (1:20; Agilent, M077501-2), as a marker for activated cells. MHCII marker genes are specifically expressed in the Micl gene signature. $^{12}$ Thus, we decided to combine these primary antibodies to visualize Micl cells as performed in the study of Mathys et al. ${ }^{12}$ as well as to estimate the proportion of cells of this particular subpopulation. Then, we incubated sections for 1 hour at room temperature with secondary antibodies $(1: 1,000)$, which were conjugated with Alexa Fluor 488 and Alexa Fluor 555 (Invitrogen, Carlsbad, CA). Cell nuclei visualized with Hoechst 33258 (Life Technologies). Sections were mounted with Shandon Immu-Mount (Thermo Fisher Scientific). We acquired all the images with a Leica TCS SP5 Confocal Laser Scanning Microscope, with a $63 \times$ objective.

\section{Quantification analysis}

We obtained full-section immunohistochemistry (IHC) images with Pannoramic MIDI II (3DHistech). Blinded to the neuropathologic diagnoses, we delimited 6 gray matter areas of the motor cortex from each case, and for each gray matter area, we randomly generated 6 regions of interest (ROIs) with the same size using the multicrop.m script. We used the Ibal.m script to quantify densities for Ibal staining in each ROI, which represents a total of 36 images per individual. This algorithm binarizes the random images and compute densities of protein expression to quantify the number of immunoreactive objects. Both in-house algorithms can be freely accessed at github.com/MemoryUnitSantPau. We used MATLAB R2017b software to develop the algorithm (The MathWorks Inc., Natick, MA). For pTDP43 quantification, 2 different researchers blinded to neuropathologic diagnoses quantified immunoreactive positive objects manually. We evaluated immunofluorescence using FIJI imaging software. We estimated an automated threshold to create binary images. Following the noise removal with the despeckle and the remove outliers' filters, we determined the number of Iba1+ microglial cells expressing MHC class II markers using the analyze particles function. 


\section{Statistical analysis}

We used the Shapiro-Wilk test to test for deviation from a standard distribution. For correlation analyses, we determined the Pearson or Spearman correlation coefficients using the ggscatter function within the ggpubr package in R. We performed mean comparison analyses through the Student $t$ test or Student test with Welch correction (using t.test function in R), depending on data distribution. Statistical significance for all tests was set at 5\% $(\alpha=0.05)$, and all statistical tests were 2 sided. We used $\mathrm{R}$ version 3.6.2 to perform all statistical analyses.

\section{Standard protocol approvals, registrations, and patient consents}

The Sant Pau Hospital Ethics Committee approved the study with all experiments with human tissue performed in accordance with the Declaration of Helsinki. The NTB of the Biobanc-Hospital Clínic-IDIBAPS supplied all tissue following approval from their Scientific Advisory Committee.

\section{Data availability statement}

We have deposited all raw sequencing data (FASTQ files) at the European Genome-phenome Archive (EGA), which is hosted by the EBI and the CRG under accession number EGAS00001004286.

\section{Results}

\section{Demographics, clinical features, and sample composition}

We performed RNAseq analysis of postmortem motor cortex samples from 11 ALS cases ( 6 females) and 8 individuals without neurologic disease ( 4 females). Postmortem interval and RIN did not differ between groups $(p=0.68$ and $p=$ 0.089 , respectively). The mean age at death was lower for ALS cases compared with HC (63.4 years; range: 54-82 years in ALS and 73.9 years; range: $58-83$ years in $\mathrm{HC}, p=$ 0.04). Patients had an average disease onset of 60.9 years (range: 46-81 years) and a disease duration of 29.9 months (range: $1-72$ months). Nine of the 11 cases $(81.8 \%)$ presented a limb disease onset (table 1). We used RNAseq data to rule out the presence of other ALS-disease causing mutations, confirming the presumably sporadic nature of our ALS group of cases.

\section{Gene expression alterations and differential isoform usage}

Differential gene expression between patients with ALS and HCs disclosed a total of 108 upregulated and 16 downregulated genes (adjusted $p<0.05$ ) (figure $1 \mathrm{~A}$ and table e-2, links.lww.com/NXI/A280). We validated 9 of the 10 genes selected by qPCR, as we did not confirm the CHI3L2 gene overexpression in patients with ALS $(p=0.085)$ (figure $1 \mathrm{~B})$. Correlation analyses between counts derived from total RNAseq data and qPCR expression values indicated a high and significant concordance between both expression measures in these 10 genes (table e-3, links.lww.com/NXI/ A280). GO and KEGG pathway analyses revealed an enriched involvement of the inflammatory response in ALS (figure 1C). The assessment of changes at the isoform expression level resulted in 167 upregulated and 40 downregulated isoforms, of which 181 were from unique genes (table e-4, links. lww.com/NXI/A280). Enrichment analyses revealed the involvement of postsynaptic density and immune response as dominant pathways (figure e-1, links.lww.com/NXI/A279). To further characterize and confirm the synaptic involvement, we used the SynGO database, which provides an expertcurated resource for synaptic function and gene enrichment analysis. ${ }^{23}$ This analysis confirmed the enrichment of postsynaptic components in our list of differentially expressed isoforms (table e-5, links.lww.com/NXI/A280). Among the list of altered genes identified by the differential isoform expression and gene expression models, 32 of them overlapped between both approaches (table e-6, links.lww.com/NXI/ A280). The involvement of synaptic-related changes was confirmed from the differential isoform usage analysis (figure e-2, links.lww.com/NXI/A279), whereas the enrichment of inflammatory markers was evidenced in the gene model approach (figure e-3, links.lww.com/NXI/A279).

\section{Cell type deconvolution}

To characterize the variability of cell types and compare cellular proportions between groups, we applied the recently developed $\mathrm{MuSiC}$ algorithm. As reference, we used data from the first and most comprehensive human snRNAseq study performed in brain tissue of individuals with a neurodegenerative disease, which includes 24 patients with Alzheimer disease and 24 HCs. ${ }^{12}$ Cell type deconvolution revealed an overrepresentation of microglial cells in the ALS motor cortex compared with HC $(p=0.025$, fold change $=1.65)$ and a reduction in excitatory neurons $(p=0.101$, fold change $=$ -0.85 ) (figure 2). We confirmed microglial upregulation using 2 additional independent reference data sets. ${ }^{10,11}$ In both cases, we found the same pattern of downregulation of excitatory neurons (figures e-4 and e-5, links.lww.com/NXI/ A279). To further gain insight into which microglial subpopulation might be driving this effect, we estimated the proportion of each subpopulation identified in the ROSMAP study. Our results showed that a specific microglial subpopulation (Mic1, as named in the study performed by Mathys and collaborators and recognized as the human $\left.\mathrm{DAM}^{12}\right)$ is disproportionately presented in the ALS motor cortex $\left(p=6.6 \times 10^{-3}\right.$, fold change $\left.=4.33\right)$ (figure 2). Among the 77 marker genes, which characterize the Micl subcluster as DAM (Micl unique genes or those overlapping with DAM), ${ }^{12} 22$ of them (28.6\%) are genes differentially expressed in our bulk RNAseq analysis (unadjusted $p<0.05$; (table e-7, links.lww.com/NXI/A280). Of interest, the proportion of Micl cells showed a positive correlation with the expression of TREM2 $\left(p=1.6 \times 10^{-3} ; R=0.67\right.$; figure e-6, links.lww.com/NXI/A279), a receptor required for the transition from the homeostatic microglia to DAM. 
A

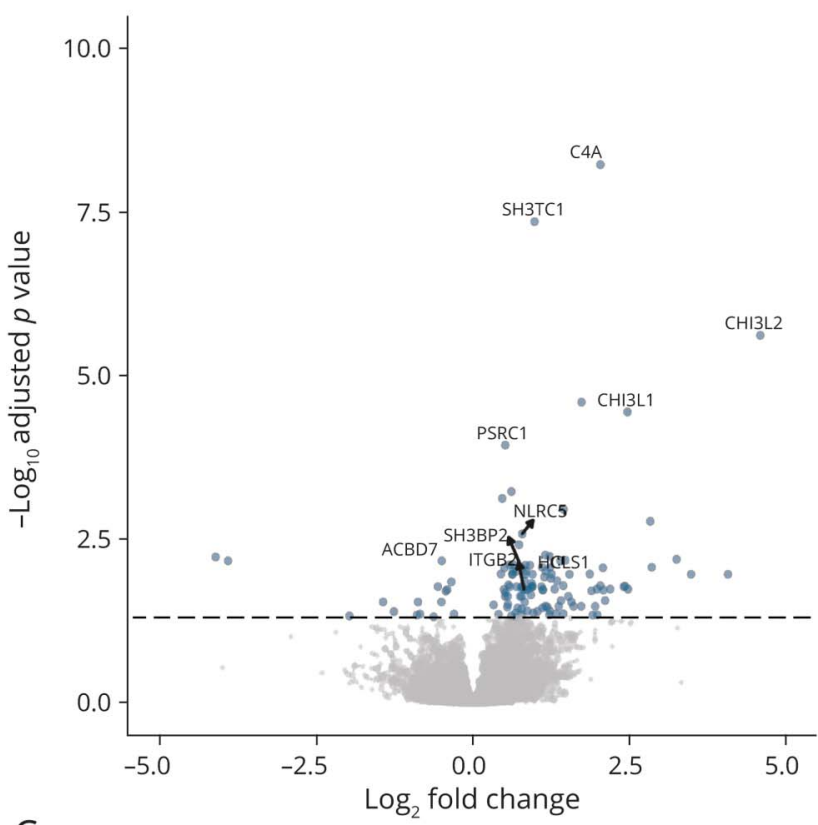

B
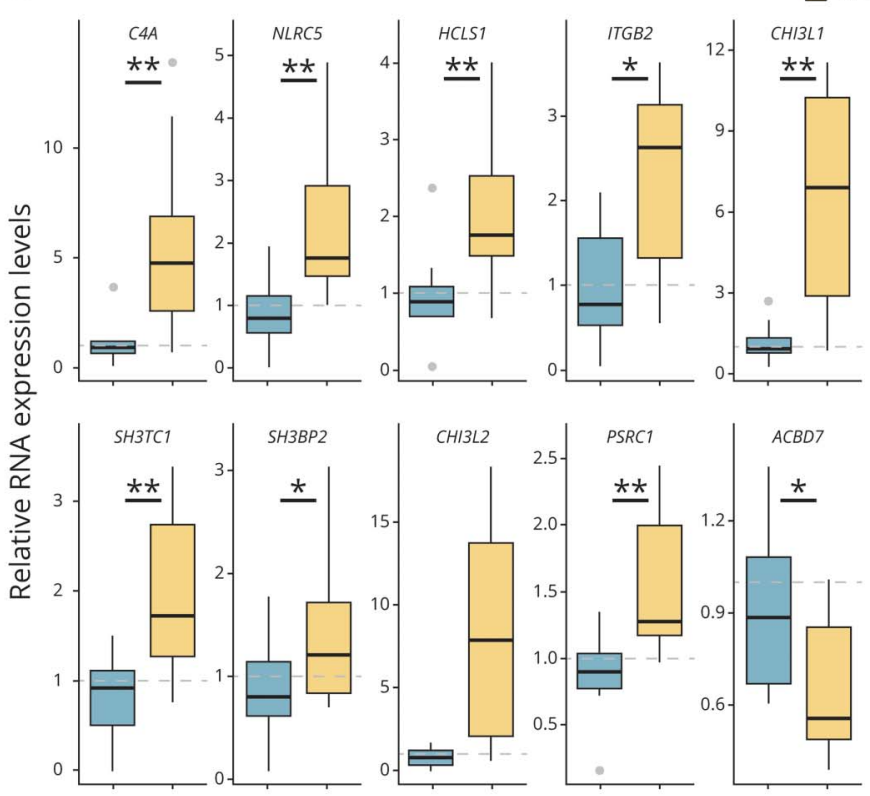

C

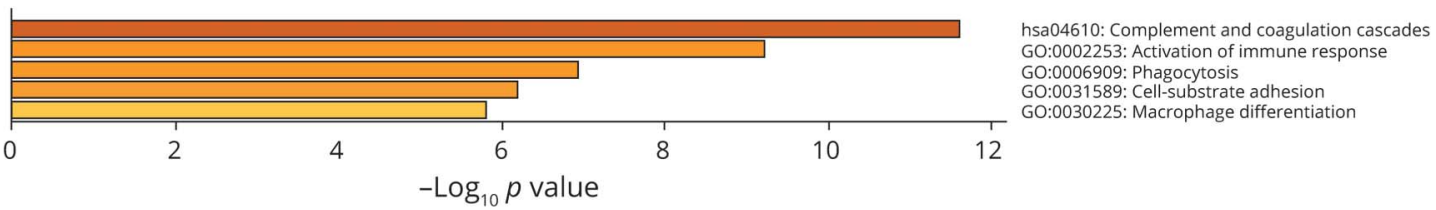

(A) Volcano plot displaying differentially expressed genes between the ALS and the HC motor cortex. The vertical axis (y-axis) corresponds to the -log 10 adjusted $p$ value, and the horizontal axis ( $\mathrm{x}$-axis) represents the $\log _{2}$ fold change value obtained when comparing ALS with $\mathrm{HC}$ gene expression. Significantly differential expressed genes are depicted with blue circles (adjusted $p$ value $<0.05$ ), whereas gray circles display the nonsignificant genes. Gene names are shown for the 10 genes selected for qPCR validation. (B) Box plot showing the relative RNA expression values in ALS and HC obtained through qPCR for the 10 genes selected for validation. Dashed line corresponds to reference relative gene expression. ${ }^{\star} p<0.05 ;{ }^{*} p<0.01$. (C) Top 5 gene ontology and KEGG pathway enrichment analyses obtained from the list of genes showing a significant differential expression. ALS = amyotrophic lateral sclerosis; $\mathrm{HC}=$ healthy control.

\section{Gene coexpression network analyses}

To identify gene clusters with varying coexpression patterns that could behave differently between patients and healthy individuals, and elucidate possible biological mechanisms driving pathologic processes, we performed a gene coexpression network analyses using the WGCNA package. The analysis disclosed a total of 8 modules. Among them, 3 modules associated with disease status (MEblack, $p=0.003 ; R$ $=0.64$; MEyellow, $p=0.025 ; R=0.57$; and MEpink, $p=$ $0.011 ; R=-0.51$; figure 3). Moreover, MEblack and MEyellow were enriched for inflammatory responses, whereas MEpink had an overrepresentation of genes involved in synaptic and neuronal functions (table e-8, links.lww.com/ NXI/A280). The SynGO database confirmed the postsynaptic signature of this enrichment in MEpink (table e-9, links.lww.com/NXI/A280). Of interest, the 2 inflammatory gene coexpression modules (MEblack and MEyellow) showed a strong negative correlation with the postsynaptic module (MEpink) $\left(p=8 \times 10^{-5} ; R=-0.78\right.$ and $p=3 \times 10^{-4}$; $R=-0.74$, respectively) (figure 3 ), suggesting that both inflammation and synaptic alterations are interconnected in ALS pathologic processes. Furthermore, the proportion of Micl cells showed a strong direct correlation with the 2 inflammatory gene coexpression modules associated with ALS (MEblack; $p=2.7 \times 10^{-8}, R=0.92$; and MEyellow; $p=1.4 \times$ $\left.10^{-3}, R=0.68\right)$ and a negative correlation with the synaptic module (MEpink; $p=2.4 \times 10^{-4}, R=-0.75$ ) (figure 3). Overall, our results from differential gene and isoform expression, gene coexpression modules, and cell type deconvolution comparisons strongly indicate that microglia-related inflammatory changes, mainly driven by the Micl subpopulation (also known as DAM), are central in ALS pathophysiology and that these inflammatory processes are closely related to the synaptic disturbances that are present in the motor cortex.

\section{Immunohistochemistry}

To further investigate the inflammatory response in postmortem brain tissue, we performed IHC analyses using Ibal, a protein whose expression is primarily restricted to homeostatic 


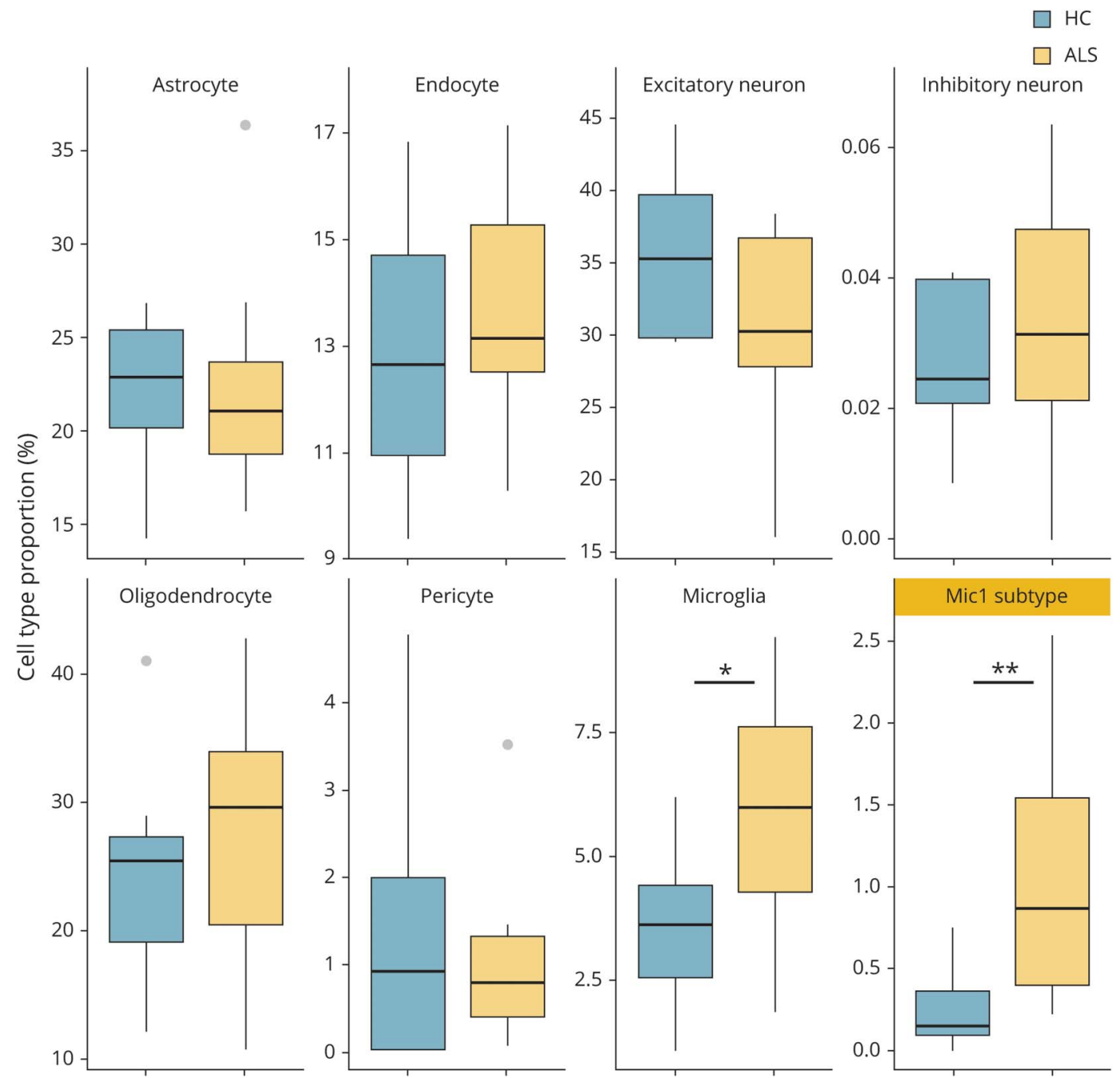

Box plot showing major cell type and Mic1 proportions for HC and ALS estimated by MuSiC using the ROSMAP human single-nucleus RNAseq data. * $p<0.05$; $* \star p<0.01$. ALS = amyotrophic lateral sclerosis; HC = healthy control; MuSiC = Multi-Subject Single Cell deconvolution; ROSMAP = Religious Orders Study and Memory and Aging Project.

microglia, to examine the broad population of microglial cells, and MHC class II, a marker responsible for antigen recognition and the activation of the adaptive immune system, which is expressed in the Micl cell subpopulation. Positive immunostaining was increased in the ALS motor cortex for Ibal; however, differences between patients and controls did not reach statistical significance ( $p=0.106$; figure e-7, links.lww.com/ NXI/A279). Notwithstanding, co-immunofluorescence of Iba1 and MHC class II markers revealed an increase in the number of microglial cells expressing MHCII markers in ALS compared with HC (70.1\% vs $10 \%$, respectively; $p=0.021)$ (figure 4 and figure e-8, links.lww.com/NXI/A279), strengthening our results and suggesting that Micl is driving neuroinflammation in the ALS motor cortex.

We finally assessed the frequency of pTDP43 immunoreactive structures in the motor cortex. As expected, patients with ALS showed a higher density of pTDP43 inclusions compared with HCs $(p=0.017)$ (figure e-9A, links.lww.com/ NXI/A279). Of interest, a high degree of variability on the number of pTDP43 aggregates was noted in ALS and was not explained by age at death, age at onset, or disease duration. The burden of pTDP43 inclusions inversely correlated with the proportion of microglial cells in our group of patients with $\operatorname{ALS}(p=0.0026 ; R=-0.81)$ (figure e-9B, links.lww.com/ NXI/A279), and the same pattern was found with the microglial subpopulation Micl $(p=0.019 ; R=-0.69)$.

\section{Discussion}

The motor cortex is one of the major vulnerable and early affected regions in ALS and represents a target region to disentangle key pathologic processes in this neurodegenerative disorder. To date, few studies have performed a whole transcriptomic assessment of the CNS in ALS. ${ }^{25-27}$ Through 

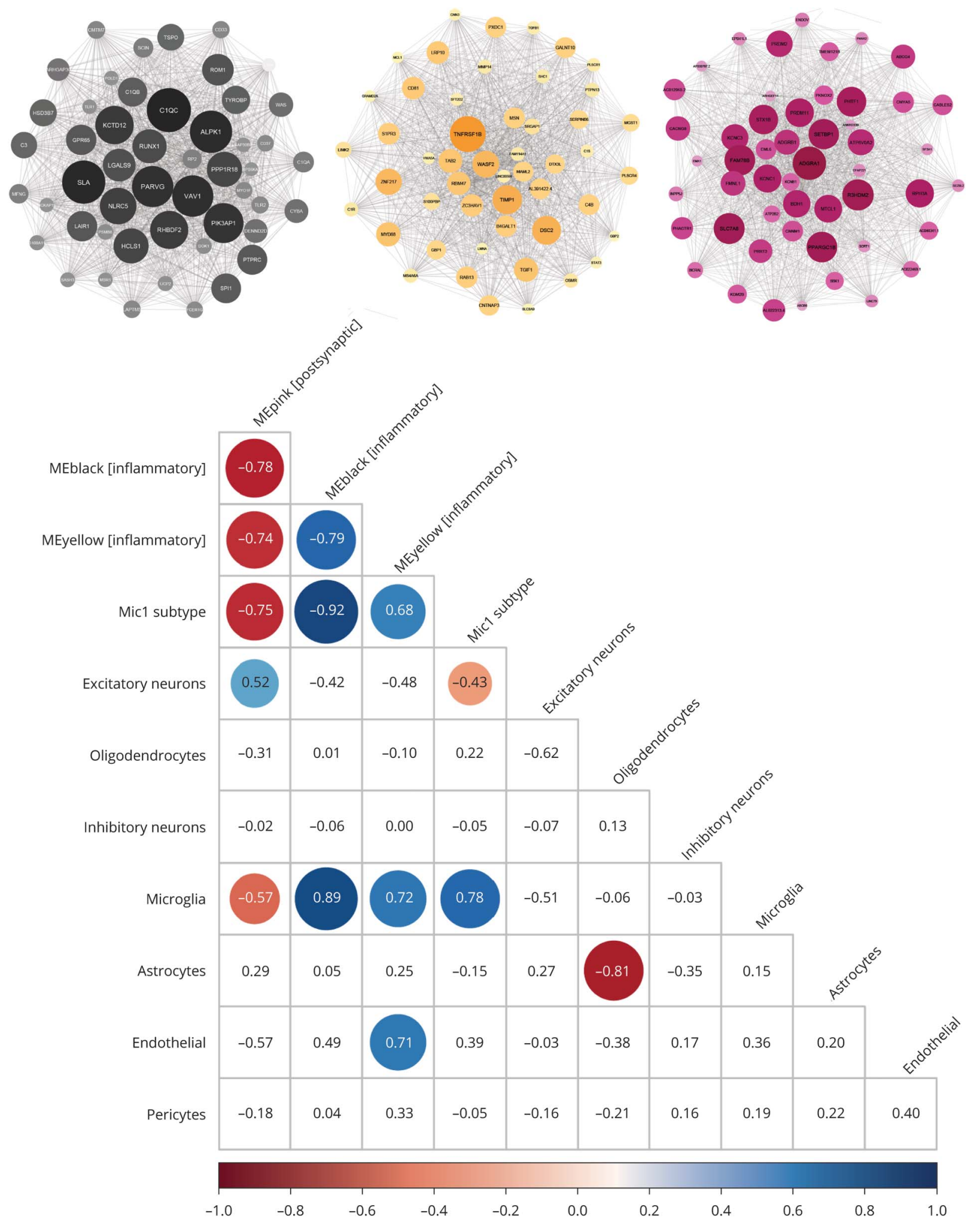

Network diagrams showing the 50 most connected genes for each of the 3 modules associated with ALS (MEblack, MEyellow, and MEpink) are depicted on the top of the figure. Node darkness and size are proportional to the number of connections within the module. Correlation matrix showing the correlation coefficients between cell type proportions is depicted for the 6 major cell types and the Mic1 (DAM) cell subtype. The 3 significant modules (MEblack, MEyellow, and MEpink), each of one sharing unique groups of coexpressed genes that are differentially expressed between patients with ALS and controls, are also included in the correlogram. Only significant correlations are depicted with a colored circle (blue for direct and red for inverse correlations). The size of the circle is proportional to the correlation significance. The plot indicates the high correlation between the 3 modules and how these modules are related to the proportion of specific cell types. ALS = amyotrophic lateral sclerosis; DAM = disease-associated microglia; HC = healthy control; WGGNA = weighted gene coexpression correlation network analyses. 

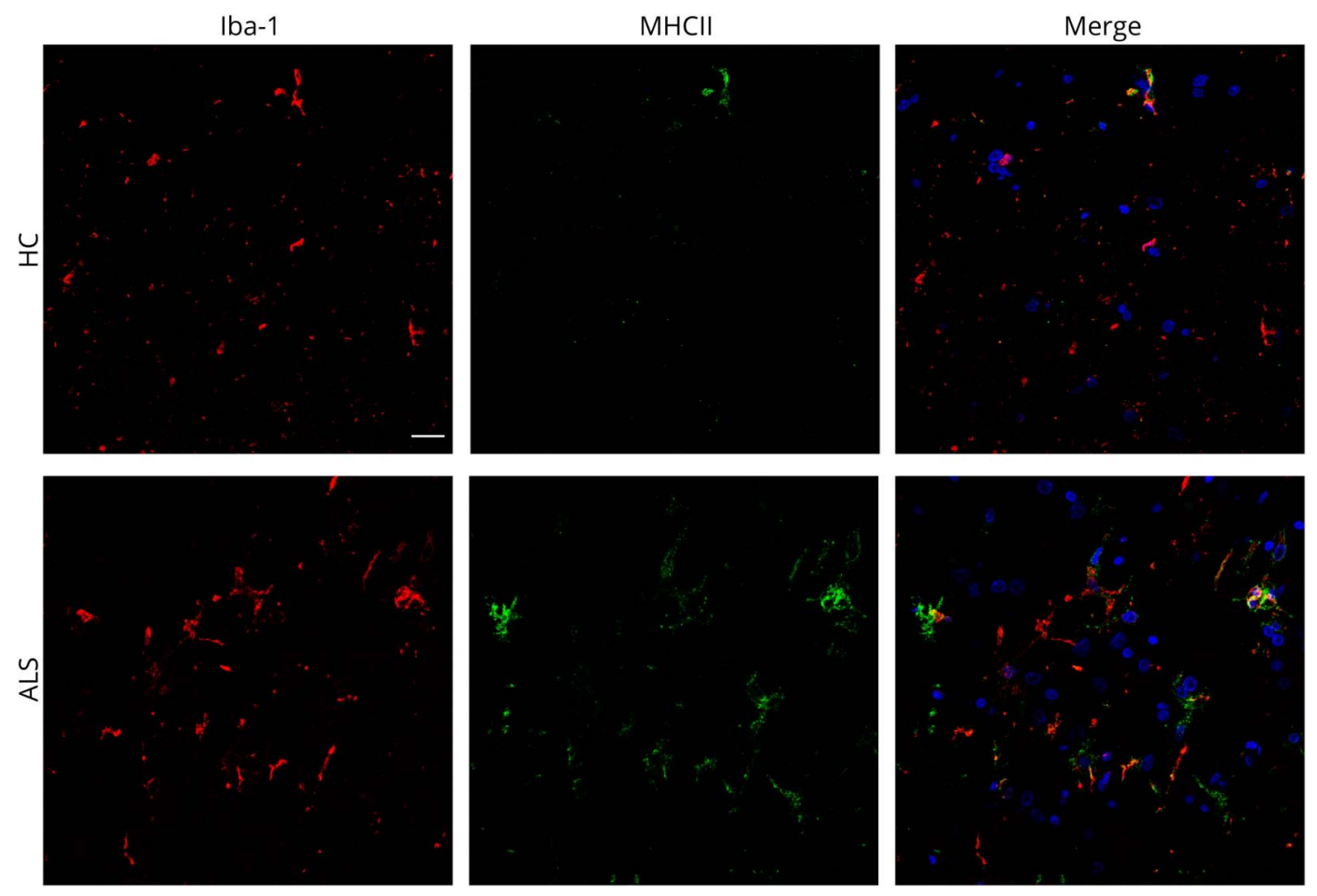

Immunohistochemistry with anti-IBA1 (red) and anti-MHC class II (green) antibodies in the ALS and HC motor cortex. Scale bar corresponds to $50 \mu \mathrm{m}$. ALS = amyotrophic lateral sclerosis; $\mathrm{HC}=$ healthy control.

an unbiased transcriptomic analysis using high-throughput RNA sequencing, we have elucidated gene and isoform expression alterations, gene coexpression networks, and cell type proportions associated with ALS. For the first time in the ALS field, we have performed cell type deconvolution using human brain single-nucleus RNA sequencing data from 3 independent sources as reference, thus providing highly reliable results that have been reinforced through our immunohistochemical analyses.

We report a list of 124 genes differentially expressed in the ALS motor cortex, which reflect the RNA expression changes in this brain region. Among them, chitinase-related genes CHI3L1 and CHI3L2 presented 2 of the most prominent shifts in gene expression, whereas CHIT1 showed a nominal significance. Of interest, these chitinases are neuroinflammatory biomarkers, which have been shown to be consistently increased in the CSF of patients with ALS compared with neurologically healthy individuals. ${ }^{7,8}$ These results indicate that among the unbiased signature of RNA alterations provided herein, some of them might lead to the discovery of novel promising biomarkers.

An exacerbated innate immune response with microgliosis has been recently described in the ALS motor cortex. ${ }^{28} \mathrm{~A}$ recent study that has investigated the whole transcriptome of the ALS motor cortex suggested that among ALS cases, a subgroup of them is characterized by a molecular signature related to glial activation and inflammation. ${ }^{27}$ Our results clearly reinforce the idea of these inflammatory-related changes in the human motor cortex and emphasize the role of DAM as a key factor in this process. First, differential gene and isoform expression data point toward an inflammatory response as a major event that is strongly intensified in the ALS motor cortex. Second, the assembly of weighted gene coexpression networks resulted in 2 significant modules associated with ALS (MEblack and MEyellow), both highly enriched with genes related to inflammatory functions. Third, cell type deconvolution of the bulk RNAseq data demonstrated an increased proportion of microglial cells compared with the motor cortex of healthy cases. In this context, studies in mice have recently evidenced DAM as the microglial subpopulation with a more prominent role in ALS. ${ }^{4}$ Our results show that Micl, the human microglial subpopulation that harbors the majority of markers found in the previously described DAM transcriptomic signature, ${ }^{12}$ is the main microglial subpopulation that drives microgliosis in ALS. In fact, almost a third of Micl (DAM) marker genes are deregulated in the motor cortex of our group of patients with ALS. Of note, Micl proportion highly correlated with the expression of TREM2, which is required to enhance the proinflammatory stage of DAM. ${ }^{4}$

Our immunohistochemical analyses did not show a relevant increased density of Ibal (a widely used marker of microglial processes) in ALS-related brain tissue, nor was the expression 
of its coding gene (AIF1) altered in our RNASeq data set. Previous studies have frequently provided contradictory results when using this marker to assess microgliosis. ${ }^{29}$ This somewhat unexpected result could be explained by the fact that some marker genes might downregulate on microglial activation. Also, whereas cell type deconvolution is performed using a complete catalogue of gene expression profiles obtained from brain-derived snRNAseq, immunohistochemistry only uses a single marker and does not reflect the complexity and heterogeneity of this cell type, making it an underpowered method to detect differences in the proportion of cell subpopulations. That being said, we did validate the increased proportion of Micl cells in the ALS motor cortex through co-immunofluorescence of 2 markers (Ibal and MHC class II) previously shown to characterize this microglial subpopulation. ${ }^{12}$ This finding further establishes Mic1 (the human disease-associated microglia) as the microglial subpopulation that drives microgliosis in the ALS motor cortex.

We observed a high degree of variability in the density of pTDP43 inclusions across patients, which could not be explained by any of the demographic or clinical features available in this study. Notably, we found a striking inverse correlation between the proportion of microglial cells and the amount of pTDP43 aggregates. These results are in line with recent in vivo and in vitro studies, suggesting that although microgliosis arises as a phagocytic response to pTDP43 aggregates, at some point, these cells lose their ability to clear these neuropathologic insults and are downregulated. ${ }^{30,31}$ Whether biofluid levels of microglial markers, such as TREM2, could be used as a proxy of pTDP43 density in the motor cortex is an avenue worth pursuing and would be a valuable addition to the biomarker arsenal for use in designing clinical trials and assessing therapeutic efficacy.

Synaptic dysfunction is an early pathogenic event in ALS. ${ }^{32}$ Our data point toward an underrepresentation of postsynaptic markers and a decrease of excitatory neurons in patients with ALS. Together, these results are consistent with upper motor neuron degeneration occurring in this brain region. A limitation of our approach is the lack of an available motor cortex snRNAseq data set, precluding any firm and more detailed conclusion related to the alteration of Betz cells, a unique class of motor neurons expressed in this specific brain area. Recent studies in other neurodegenerative diseases have suggested that microglia are a key and early mediator of synapse loss through phagocytosis induced by the complement cascade. ${ }^{33,34}$ Our gene expression data show an increased expression of some key complement cascade-related genes and the most significant enriched pathway corresponds to the complement and coagulation cascades, reinforcing this hypothesis. Furthermore, our results indicate that the 2 neuroinflammatory-related modules of gene expression (MEblack and MEyellow) and the proportion of Micl cells inversely correlate with the presence of synaptic markers.
Overall, our study strongly suggests that DAM plays a key role in driving neuroinflammatory changes and synapse loss in the ALS motor cortex. The identification of specific microglial populations with well-defined transcriptional signatures will contribute to disentangle new mechanisms and novel therapeutic targets to fight against this devastating disorder.

\section{Acknowledgment}

The authors are indebted to the IDIBAPS Biobank for sample and data procurement.

\section{Study funding}

The authors are indebted to the "Fundación Española para el Fomento de la Investigación de la Esclerosis Lateral Amiotrófica (FUNDELA)" for funding the present study. O. Dols-Icardo is a recipient of a grant by The Association for Frontotemporal Degeneration (Clinical Research Postdoctoral Fellowship, AFTD 2019-2021). This work was supported by research grants from Institute of Health Carlos III (ISCIII), Spain PI18/00326 to J.C., PI18/00435 to D.A., and INT19/00016 to D.A., and by the Department of Health Generalitat de Catalunya PERIS program SLT006/17/125 to D.A. This work was also supported in part by Generalitat de Catalunya (2017 SGR 00547) to the "Grup de Recerca en Demències: Sant Pau."

\section{Disclosure}

The authors report no disclosures relevant to the manuscript. Go to Neurology.org/NN for full disclosures.

\section{Publication history}

Received by Neurology: Neuroimmunology \& Neuroinflammation February 21, 2020. Accepted in final form May 15, 2020.

\begin{tabular}{|c|c|c|}
\hline Name & Location & Contribution \\
\hline $\begin{array}{l}\text { Oriol Dols- } \\
\text { Icardo, PhD }\end{array}$ & $\begin{array}{l}\text { Sant Pau Biomedical } \\
\text { Research Institute, Hospital } \\
\text { de la Santa Creu i Sant Pau, } \\
\text { Barcelona, Spain }\end{array}$ & $\begin{array}{l}\text { Major role in the design } \\
\text { and conceptualization of } \\
\text { the study; drafted the } \\
\text { manuscript; and analyzed } \\
\text { the data }\end{array}$ \\
\hline $\begin{array}{l}\text { Víctor } \\
\text { Montal, MSc }\end{array}$ & $\begin{array}{l}\text { Sant Pau Biomedical } \\
\text { Research Institute, Hospital } \\
\text { de la Santa Creu i Sant Pau, } \\
\text { Barcelona, Spain }\end{array}$ & $\begin{array}{l}\text { Analyzed the data and } \\
\text { participated in manuscript } \\
\text { drafting }\end{array}$ \\
\hline $\begin{array}{l}\text { Sònia Sirisi, } \\
\text { PhD }\end{array}$ & $\begin{array}{l}\text { Sant Pau Biomedical } \\
\text { Research Institute, Hospital } \\
\text { de la Santa Creu i Sant Pau, } \\
\text { Barcelona, Spain }\end{array}$ & $\begin{array}{l}\text { Analyzed the data and } \\
\text { participated in manuscript } \\
\text { drafting }\end{array}$ \\
\hline $\begin{array}{l}\text { Gema } \\
\text { López- } \\
\text { Pernas, MSc }\end{array}$ & $\begin{array}{l}\text { Sant Pau Biomedical } \\
\text { Research Institute, Hospital } \\
\text { de la Santa Creu i Sant Pau, } \\
\text { Barcelona, Spain }\end{array}$ & $\begin{array}{l}\text { Analyzed the data and } \\
\text { performed a part of the } \\
\text { experiments }\end{array}$ \\
\hline $\begin{array}{l}\text { Laura } \\
\text { Cervera- } \\
\text { Carles, PhD }\end{array}$ & $\begin{array}{l}\text { Sant Pau Biomedical } \\
\text { Research Institute, Hospital } \\
\text { de la Santa Creu i Sant Pau, } \\
\text { Barcelona, Spain }\end{array}$ & $\begin{array}{l}\text { Participated in manuscript } \\
\text { drafting and data } \\
\text { interpretation }\end{array}$ \\
\hline
\end{tabular}


Appendix (continued)

\begin{tabular}{|c|c|c|}
\hline Name & Location & Contribution \\
\hline $\begin{array}{l}\text { Marta } \\
\text { Querol- } \\
\text { Vilaseca, } \\
\text { MSc }\end{array}$ & $\begin{array}{l}\text { Sant Pau Biomedical } \\
\text { Research Institute, Hospital } \\
\text { de la Santa Creu i Sant Pau, } \\
\text { Barcelona, Spain }\end{array}$ & $\begin{array}{l}\text { Participated in manuscript } \\
\text { drafting and data } \\
\text { interpretation }\end{array}$ \\
\hline $\begin{array}{l}\text { Laia Muñoz, } \\
\text { BSc }\end{array}$ & $\begin{array}{l}\text { Sant Pau Biomedical } \\
\text { Research Institute, Hospital } \\
\text { de la Santa Creu i Sant Pau, } \\
\text { Barcelona, Spain }\end{array}$ & $\begin{array}{l}\text { Contributed to sample } \\
\text { preparation and } \\
\text { participated in } \\
\text { experimental procedures }\end{array}$ \\
\hline $\begin{array}{l}\text { Olivia } \\
\text { Belbin, PhD }\end{array}$ & $\begin{array}{l}\text { Sant Pau Biomedical } \\
\text { Research Institute, Hospital } \\
\text { de la Santa Creu i Sant Pau, } \\
\text { Barcelona, Spain }\end{array}$ & $\begin{array}{l}\text { Participated in the revision } \\
\text { and editing of the } \\
\text { manuscript and data } \\
\text { interpretation }\end{array}$ \\
\hline $\begin{array}{l}\text { Daniel } \\
\text { Alcolea, } \\
\text { MD, PhD }\end{array}$ & $\begin{array}{l}\text { Sant Pau Biomedical } \\
\text { Research Institute, Hospital } \\
\text { de la Santa Creu i Sant Pau, } \\
\text { Barcelona, Spain }\end{array}$ & $\begin{array}{l}\text { Participated in data } \\
\text { collection, analysis, and } \\
\text { data interpretation }\end{array}$ \\
\hline $\begin{array}{l}\text { Laura } \\
\text { Molina- } \\
\text { Porcel, MD, } \\
\text { PhD }\end{array}$ & $\begin{array}{l}\text { Neurological Tissue Bank } \\
\text { of the Biobanc-Hospital } \\
\text { Clínic-IDIBAPS, Barcelona, } \\
\text { Spain }\end{array}$ & $\begin{array}{l}\text { Contributed to sample } \\
\text { preparation and } \\
\text { participated in } \\
\text { experimental procedures }\end{array}$ \\
\hline $\begin{array}{l}\text { Jordi } \\
\text { Pegueroles, } \\
\text { MSc }\end{array}$ & $\begin{array}{l}\text { Sant Pau Biomedical } \\
\text { Research Institute, Hospital } \\
\text { de la Santa Creu i Sant Pau, } \\
\text { Barcelona, Spain }\end{array}$ & $\begin{array}{l}\text { Participated in data } \\
\text { analysis }\end{array}$ \\
\hline
\end{tabular}

\begin{tabular}{|c|c|c|}
\hline $\begin{array}{l}\text { Janina } \\
\text { Turón-Sans, } \\
\text { MD }\end{array}$ & $\begin{array}{l}\text { Sant Pau Biomedical } \\
\text { Research Institute, Hospital } \\
\text { de la Santa Creu i Sant Pau, } \\
\text { Barcelona, Spain }\end{array}$ & $\begin{array}{l}\text { Major role in the } \\
\text { acquisition of samples and } \\
\text { data and reviewed and } \\
\text { edited the manuscript }\end{array}$ \\
\hline $\begin{array}{l}\text { Rafael } \\
\text { Blesa, MD, } \\
\text { PhD }\end{array}$ & $\begin{array}{l}\text { Sant Pau Biomedical } \\
\text { Research Institute, Hospital } \\
\text { de la Santa Creu i Sant Pau, } \\
\text { Barcelona, Spain }\end{array}$ & $\begin{array}{l}\text { Major role in the } \\
\text { acquisition of samples and } \\
\text { data and reviewed and } \\
\text { edited the manuscript }\end{array}$ \\
\hline $\begin{array}{l}\text { Alberto } \\
\text { Lleó, MD, } \\
\text { PhD }\end{array}$ & $\begin{array}{l}\text { Sant Pau Biomedical } \\
\text { Research Institute, Hospital } \\
\text { de la Santa Creu i Sant Pau, } \\
\text { Barcelona, Spain }\end{array}$ & $\begin{array}{l}\text { Major role in the } \\
\text { acquisition of samples and } \\
\text { data and reviewed and } \\
\text { edited the manuscript }\end{array}$ \\
\hline $\begin{array}{l}\text { Juan Fortea, } \\
\text { MD, PhD }\end{array}$ & $\begin{array}{l}\text { Sant Pau Biomedical } \\
\text { Research Institute, Hospital } \\
\text { de la Santa Creu i Sant Pau, } \\
\text { Barcelona, Spain }\end{array}$ & $\begin{array}{l}\text { Major role in the } \\
\text { acquisition of samples and } \\
\text { data and reviewed and } \\
\text { edited the manuscript }\end{array}$ \\
\hline $\begin{array}{l}\text { Ricard } \\
\text { Rojas- } \\
\text { García, MD, } \\
\text { PhD }\end{array}$ & $\begin{array}{l}\text { Sant Pau Biomedical } \\
\text { Research Institute, Hospital } \\
\text { de la Santa Creu i Sant Pau, } \\
\text { Barcelona, Spain }\end{array}$ & $\begin{array}{l}\text { Major role in the } \\
\text { acquisition of samples and } \\
\text { data and reviewed and } \\
\text { edited the manuscript }\end{array}$ \\
\hline $\begin{array}{l}\text { Jordi } \\
\text { Clarimón, } \\
\text { PhD }\end{array}$ & $\begin{array}{l}\text { Sant Pau Biomedical } \\
\text { Research Institute, Hospital } \\
\text { de la Santa Creu i Sant Pau, } \\
\text { Barcelona, Spain }\end{array}$ & $\begin{array}{l}\text { Major role in funding } \\
\text { acquisition; design and } \\
\text { conceptualization of the } \\
\text { study; drafted the } \\
\text { manuscript; and analyzed } \\
\text { the data }\end{array}$ \\
\hline
\end{tabular}

\section{References}

1. Brettschneider J, Del Tredici K, Toledo JB, et al. Stages of pTDP-43 pathology in amyotrophic lateral sclerosis. Ann Neurol 2013;74:20-38.

2. Liu J, Wang F. Role of neuroinflammation in amyotrophic lateral sclerosis: cellular mechanisms and therapeutic implications. Front Immunol 2017;8:1005.

3. Prinz M, Jung S, Priller J. Microglia biology: one century of evolving concepts. Cell 2019;179:292-311.

4. Keren-Shaul H, Spinrad A, Weiner A, et al. A unique microglia type Associated with restricting development of Alzheimer's disease. Cell 2017;169:1276-1290.
5. Steinacker P, Verde F, Fang L, et al. Chitotriosidase (CHIT1) is increased in microglia and macrophages in spinal cord of amyotrophic lateral sclerosis and cerebrospinal fluid levels correlate with disease severity and progression. J Neurol Neurosurg Psychiatry 2018;89:239-247.

6. Thompson AG, Gray E, Thézénas ML, et al. Cerebrospinal fluid macrophage biomarkers in amyotrophic lateral sclerosis. Ann Neurol 2018;83:258-268.

7. Illán-Gala I, Alcolea D, Montal V, et al. CSF sAPP $\beta$, YKL-40, and NfL along the ALS FTD spectrum. Neurology 2018;91:e1619-e1628.

8. Thompson AG, Gray E, Bampton A, Raciborska D, Talbot K, Turner MR. CSF chitinase proteins in amyotrophic lateral sclerosis. J Neurol Neurosurg Psychiatry 2019;90:1215-1220.

9. Ling SC, Polymenidou M, Cleveland DW. Converging mechanisms in ALS and FTD: disrupted RNA and protein homeostasis. Neuron 2013;79:416-438.

10. Hodge RD, Bakken TE, Miller JA, et al. Conserved cell types with divergent features in human versus mouse cortex. Nature 2019;573:61-68

11. Lake BB, Chen S. Sos BC Integrative single-cell analysis of transcriptional and epigenetic states in the human adult brain. Nat Biotechnol 2018;36:70-80.

12. Mathys H, Davila-Velderrain J, Peng Z, et al. Single-cell transcriptomic analysis of Alzheimer's disease. Nature 2019;570:332-337.

13. Wang X, Park J, Susztak K, Zhang NR, Li M. Bulk tissue cell type deconvolution with multi-subject single-cell expression reference. Nat Commun 2019; $10: 380$.

14. Brooks BR, Miller RG, Swash M, Munsat TL; World Federation of Neurology Research Group on Motor Neuron Diseases. El Escorial revisited: revised criteria for the diagnosis of amyotrophic lateral sclerosis. Amyotroph Lateral Scler Other Motor Neuron Disord 2000;1:293-299.

15. García-Redondo A, Dols-Icardo O, Rojas-García R, et al. Analysis of the C9orf72 gene in patients with amyotrophic lateral sclerosis in Spain and different populations worldwide. Hum Mutat 2013;34:79-82.

16. Dols-Icardo O, García-Redondo A, Rojas-García R, et al. Analysis of known amyotrophic lateral sclerosis and frontotemporal dementia genes reveals a substantial genetic burden in patients manifesting both diseases not carrying the C9orf 72 expansion mutation. J Neurol Neurosurg Psychiatry 2018;89:162-168.

17. Dobin A, Davis CA, Schlesinger F, et al. STAR: ultrafast universal RNA-seq aligner. Bioinformatics 2013;29:15-21.

18. McKenna A, Hanna M, Banks E, et al. The Genome Analysis Toolkit: a MapReduce framework for analyzing next-generation DNA sequencing data. Genome Res 2010, 20:1297-1303.

19. Liao Y, Smyth GK, Shi W. The R package Rsubread is easier, faster, cheaper and better for alignment and quantification of RNA sequencing reads. Nucleic Acids Res 2019; 47:e47.

20. Patro R, Duggal G, Love MI, Irizarry RA, Kingsford C. Salmon provides fast and bias-aware quantification of transcript expression. Nat Methods 2017;14: 417-419.

21. Love MI, Huber W, Anders S. Moderated estimation of fold change and dispersion for RNA-seq data with DESeq2. Genome Biol 2014;15:550.

22. Zhou Y, Zhou B, Pache L, et al. Metascape provides a biologist-oriented resource for the analysis of systems-level datasets. Nat Commun 2019;10:1523.

23. Koopmans F, van Nierop P, Andres-Alonso M, et al. SynGO: an evidence-based, expert-curated knowledge base for the synapse. Neuron 2019;103:217-234.e4.

24. Langfelder P, Horvath S. WGCNA: an R package for weighted correlation network analysis. BMC Bioinformatics 2008;9:559.

25. Prudencio M, Belzil VV, Batra R, et al. Distinct brain transcriptome profiles in C9orf72-associated and sporadic ALS. Nat Neurosci 2015;18:1175-1182.

26. D’Erchia AM, Gallo A, Manzari C, et al. Massive transcriptome sequencing of human spinal cord tissues provides new insights into motor neuron degeneration in ALS. Sci Rep 2017;7:10046.

27. Tam OH, Rozhkov NV, Shaw R, et al. Postmortem cortex samples identify distinct molecular subtypes of ALS: retrotransposon activation, oxidative stress, and activated glia. Cell Rep 2019;29:1164-1177.e5.

28. Jara JH, Genç B, Stanford MJ, et al. Evidence for an early innate immune response in the motor cortex of ALS. J Neuroinflammation 2017;14:129.

29. Hopperton KE, Mohammad D, Trépanier MO, Giuliano V, Bazinet RP. Markers of microglia in post-mortem brain samples from patients with Alzheimer's disease: a systematic review. Mol Psychiatry 2018;23:177-198.

30. Spiller KJ, Restrepo CR, Khan T, et al. Microglia-mediated recovery from ALSrelevant motor neuron degeneration in a mouse model of TDP-43 proteinopathy. Nat Neurosci 2018;21:329-340.

31. Svahn AJ, Don EK, Badrock AP, et al. Nucleo-cytoplasmic transport of TDP43 studied in real time: impaired microglia function leads to axonal spreading of TDP-43 in degenerating motor neurons. Acta Neuropathol 2018;136: 445-459.

32. Henstridge CM, Pickett E, Spires-Jones TL. Synaptic pathology: a shared mechanism in neurological disease. Ageing Res Rev 2016;28:72-84.

33. Hong S, Beja-Glasser VF, Nfonoyim BM, et al. Complement and microglia mediate early synapse loss in Alzheimer mouse models. Science 2016;352:712-716.

34. Dejanovic B, Huntley MA, De Mazière A, et al. Changes in the synaptic proteome in tauopathy and rescue of tau-induced synapse loss by C1q antibodies. Neuron 2018; 100:1322-1336.e7. 


\section{Neurology \\ Neuroimmunology \& Neuroinflammation}

\section{Motor cortex transcriptome reveals microglial key events in amyotrophic lateral sclerosis}

Oriol Dols-Icardo, Víctor Montal, Sònia Sirisi, et al.

Neurol Neuroimmunol Neuroinflamm 2020;7;

DOI 10.1212/NXI.0000000000000829

This information is current as of July 15, 2020

\section{Updated Information \& Services}

References

Citations

Subspecialty Collections

Permissions \& Licensing

Reprints including high resolution figures, can be found at: http://nn.neurology.org/content/7/5/e829.full.html

This article cites 34 articles, 5 of which you can access for free at: http://nn.neurology.org/content/7/5/e829.full.html\#\#ref-list-1

This article has been cited by 3 HighWire-hosted articles: http://nn.neurology.org/content/7/5/e829.full.html\#\#otherarticles

This article, along with others on similar topics, appears in the following collection(s):

Amyotrophic lateral sclerosis

http://nn.neurology.org//cgi/collection/amyotrophic_lateral_sclerosis_ Gene expression studies

http://nn.neurology.org//cgi/collection/gene_expression_studies

Information about reproducing this article in parts (figures,tables) or in its entirety can be found online at:

http://nn.neurology.org/misc/about.xhtml\#permissions

Information about ordering reprints can be found online:

http://nn.neurology.org/misc/addir.xhtml\#reprintsus

Neurol Neuroimmunol Neuroinflamm is an official journal of the American Academy of Neurology.

Published since April 2014, it is an open-access, online-only, continuous publication journal. Copyright

Copyright $\odot 2020$ The Author(s). Published by Wolters Kluwer Health, Inc. on behalf of the American

Academy of Neurology.. All rights reserved. Online ISSN: 2332-7812.

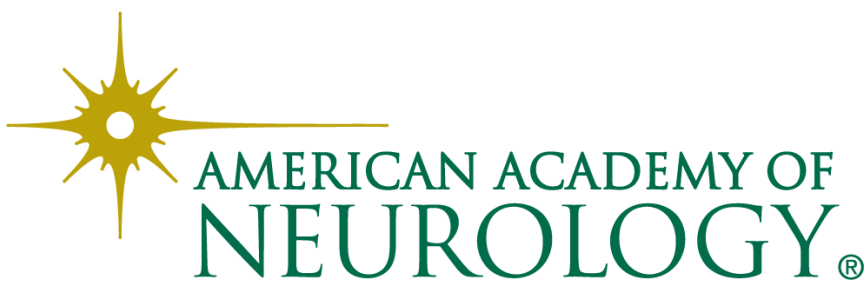

